\title{
The effect of bimodality on tensile properties of filled silicone networks
}

\author{
G. B. Shah* \\ Applied chemistry Laboratories (ACL), Pakistan Institute of Nuclear Science and Technology (PINSTECH), P.O. Nilore, \\ Islamabad, Pakistan
}

Received 22 September 2008; accepted in revised form 10 November 2008

\begin{abstract}
The effect of blending short and long chains (bimodality) silicone prepolymer, in the presence of a filler (17.2 and $30.2 \% \mathrm{w} / \mathrm{w}$ ) on tensile properties such as ultimate tensile strength (UTS), percent elongation at break (\% Eb), 100\% modulus and elastic modulus has been investigated. The content of short chain prepolymer was varied from 0-69\% and $0-58 \%$ for 17.2 and $30.2 \%$ filled silicones respectively. It was found out that the tensile properties were enhanced for the low filled (17.2\%) silicone networks while in the case of highly filled (30.2\%) silicone networks; the bimodality adversely affected the tensile properties such as UTS, \% Eb. It is also observed that the optimum in these properties was recorded at $95 \mathrm{~mol} \%$ as compared to $70 \mathrm{~mol} \%$ of short chain prepolymer for unfilled silicone system. The phenomenon of improvement in mechanical properties due to bimodality in this system has been discussed.
\end{abstract}

Keywords: material testing, bimodal polymer networks, monomodal polymer networks, mixing, crosslinking

\section{Introduction}

The blending of long and short chains of the same polymer which results in bimodal molecular weight distribution is known as bimodality and the resulting polymer networks prepared from such blends are known as bimodal networks. The interest in studies of these bimodal networks initiated due to the fact that these bimodal networks exhibits a combination of good mechanical properties, such as tear energy, tensile behaviour, resilience [1-3], non-linear stress-optic response [4-6] and a proportional orientation-stretch response with respect to molar concentration of short chains HOPDMS (hydroxy functional polydimethylsiloxane) in the bimodal network [7, 8].

The mechanism of enhancement in these properties due to bimodality is not clear [2], but the accepted view to date is that the introduction of short chains prepolymers creates high crosslink density domains with in the otherwise uniform polymer networks. This results in the low incidence of chain irregularities (i.e. the presence of polymer ends that do not form part of the load bearing networks) and limited extensibility to the polymer networks hence gives rise to the non-Gaussian effects which are reported to be responsible for the enhancement $[1,9]$. The domains of high crosslink density within the bimodal networks support the applied stress, thus hiding the imperfections which are otherwise more exposed in monomodal networks. The longer prepolymers chains which have potentials for high extension, on the other hand provide high tear energy in the unfilled and filled state, tensile properties, in accordance with the Lake-Thomas equation [10]. These effects coupled together, make bimodal polymer networks superior to monomodal ones in respect of tear properties. Tensile and shear properties have also been reported to be improved 
due to bimodality for unfilled silicone networks $[11,12]$.

A great deal of work has been done by other researchers [13-19] to prepare such tough elastomers by using this technique. The concept of bimodality is not only limited to silicone networks for enhancement in mechanical properties but, has also been reported for enhancement in system such as polyisoprene [20]. Grobler and Mcgill observed improvement in tear and tensile properties for polyisoprene via different curing condition and have attributed these improvements to the polymer network heterogeneity.

Apart from the economical reasons, fillers are being used as reinforcing agents in various polymer systems. It is this example of reinforcement of properties of expensive polymer by cheap particulate fillers that is the root of the commercial exploitation of these materials [21].

Coupling the concept of bimodality with reinforcement due to filler seemed to be a novel way of achieving enhancement in the properties of silicone networks. For this purpose, the effect of bimodality on tensile properties of the bimodal silicone networks in the presence of pyrogenic silica HDK type 2000-4 as a filler has been studied. In this respect two concentrations i.e. 17.2 and $30.2 \%$ w/w HDK, in conjunction with variation of concentration of short chain silicone prepolymer have been used for silicone networks preparation. These networks have been studied for their tensile properties.

\section{Experimental}

\subsection{Materials}

The liquid hydroxyfunctional polydimethylsiloxanes (HOPDMS) prepolymers (Silopren), long chain $(\mathrm{C} 50, \mathrm{M} . \mathrm{Wt}=88000)$ and a short chain silicone prepolymer of viscosity $100 \mathrm{mPa} \cdot \mathrm{s}$ (P100) used were supplied by Bayer Ltd. and Petrarch Ltd. respectively. The catalyst, dioctyltinmaleate (DOTM) (LT195, M.W 459), was supplied by Lankro Ltd. and a trifunctional crosslinker vinyltris(ethoxymethoxy)silane VTEMS (A172, M.W 280.4) was procured from Union Carbide Ltd. The filler employed in this study, supplied by Wacker Ltd was pyrogenic silica HDK type H 2000-4.

\subsection{Methods}

Two series of compositions having different concentrations i.e. 17.2 and $30.2 \% \mathrm{w} / \mathrm{w}$ of the filler (HDK) based on the total weight were mixed with C50 separately. The amount of short chain prepolymer i.e. P100, to be mixed with the above compositions was varied from 0 to $69 \%$ and 0 to $58 \%$ for the 17.2 and $30.2 \%$ filled HOPDMS respectively. The concentrations of crosslinker and catalyst for these compositions were kept constant i.e. 13.79 and $0.7 \%$ respectively. Each of the compositions, after mixing to a homogeneous paste, was cast onto a polyethylene plate separately and spread to a uniform film of approximately $1.4 \mathrm{~mm}$ thickness. These films were left in an open air at room temperature $\left(25^{\circ} \mathrm{C}\right)$ for seven days to complete the crosslinking (curing) reaction. Dumbbell shaped specimens were cut from the films according to BS 903 Part A2 1956 Die C. Instran Model 4301 High Wycombe (England) was used for measuring tensile properties i.e. tensile strength (TS) and percent elongation at break $(\% \mathrm{~Eb})$ at a strain rate of $50 \mathrm{~mm} / \mathrm{min}$. An average of the three modal values out of that of five specimens tested has been used for presentation.

\section{Results and discussions}

As reported [3] previously, the amount of crosslinker necessary to cure the mixture has a positive effect on the mechanical properties such as $\% \mathrm{~Eb}$, and UTS and $100 \%$ modulus. In the present studies for the filled bimodal silicone network preparation, the amount of crosslinker necessary to cure the whole series of each of the two compositions separately, was selected on the basis of trial and error experiments.

The polymer networks prepared from the two series of filled bimodal prepolymer compositions prepared were tested for tensile properties such as $\% \mathrm{~Eb}$, ultimate tensile strength (UTS), $100 \%$ modulus. The $17.2 \%$ filled silicone networks showed an increase in these tensile properties with the increases in concentration of short chain prepolymer (bimodality). However, surprisingly, when this silicone system is filled to $30.2 \% \mathrm{w} / \mathrm{w}$ level, the ultimate tensile property suffers continuous decrease 
Table 1. Variation of tensile properties of filled C50 networks with bimodality

\begin{tabular}{|c|c|c|c|c|c|c|c|c|c|c|c|}
\hline \multicolumn{2}{|c|}{$\begin{array}{c}\text { P100 } \\
\text { concentration }\end{array}$} & \multicolumn{4}{|c|}{$17.2 \%$ filled network } & \multicolumn{2}{|c|}{$\begin{array}{c}\text { P100 } \\
\text { concentration }\end{array}$} & \multicolumn{4}{|c|}{$32.2 \%$ filled networks } \\
\hline Wt\% & Mol\% & $\% \mathbf{E b}$ & $\begin{array}{c}\text { UTS } \\
{\left[\mathrm{MN} \cdot \mathbf{m}^{-2}\right]}\end{array}$ & $\begin{array}{c}100 \% \\
\text { modulus } \\
{\left[\mathrm{MN} \cdot \mathrm{m}^{-2}\right]}\end{array}$ & $\begin{array}{c}\mathbf{E} \\
{\left[\mathbf{M N} \cdot \mathbf{m}^{-2}\right]}\end{array}$ & $\mathrm{Wt} \%$ & Mol\% & $\% \mathbf{E b}$ & $\begin{array}{c}\text { UTS } \\
{\left[\mathrm{MN} \cdot \mathbf{m}^{-2}\right]}\end{array}$ & $\begin{array}{c}100 \% \\
\text { modulus } \\
{\left[\mathrm{MN} \cdot \mathbf{m}^{-2}\right]}\end{array}$ & $\begin{array}{c}\mathbf{E} \\
{\left[\mathbf{M N} \cdot \mathbf{m}^{-2}\right]}\end{array}$ \\
\hline 0 & 0.0 & 215 & 1.520 & 1.121 & 1.12 & 0 & 0.0 & 280 & 4.75 & 2.97 & 5.28 \\
\hline 20 & 64.0 & 214 & 1.631 & 1.301 & 1.17 & 11 & 52.1 & 195 & 4.17 & 3.25 & 4.76 \\
\hline 40 & 84.2 & 250 & 2.516 & 1.384 & 1.94 & 21 & 71.8 & 132 & 4.23 & 3.47 & 4.76 \\
\hline 50 & 89.9 & 251 & 2.760 & 1.567 & 2.01 & 31 & 82.9 & 120 & 3.94 & - & 5.55 \\
\hline 60 & 94.1 & 267 & 2.841 & 1.646 & 2.13 & 41 & 90.1 & 126 & 4.23 & - & 5.01 \\
\hline 64 & 95.5 & 300 & 3.152 & 1.722 & 2.41 & 51 & 95.5 & 78 & 3.24 & - & 5.34 \\
\hline 72 & 97.9 & 350 & 2.817 & 1.197 & 2.22 & 55 & 96.7 & 78 & 3.21 & - & 6.05 \\
\hline 76 & 99.0 & 298 & 2.513 & 1.173 & 2.22 & 59 & 98.1 & 80 & 2.87 & - & 5.01 \\
\hline 80 & 100.0 & 276 & 2.417 & 1.090 & 1.79 & 61 & 98.8 & 96 & 3.56 & - & 7.54 \\
\hline & & & & & & 71 & 100.0 & 68 & 3.64 & - & 6.84 \\
\hline
\end{tabular}

Crosslinker $(\mathrm{A} 172)=13.8 \% \mathrm{w} / \mathrm{w}$; Catalyst $(\mathrm{LT} 195)=0.07 \% \mathrm{w} / \mathrm{w}$.

The calculation of mole $\%$ of P100 is based on the limiting values of prepolymer networks (Mc P100 $=2250$ and Mc C50 = 12000) while the concentration of filler has been ignored.

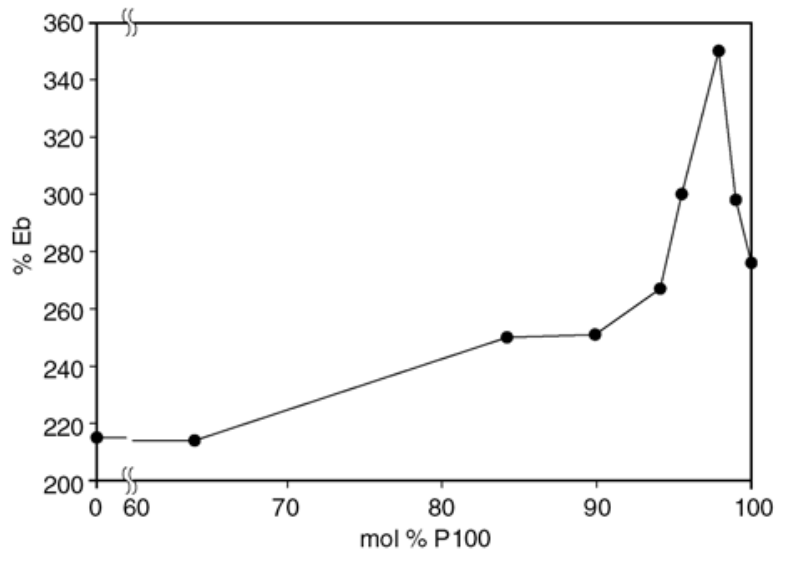

Figure 1. Effect of variation of bimodality on $\% \mathrm{~Eb}$ for $17.2 \%$ filled silicone networks

with increase in concentration of short chain prepolymer (Table 1). Previously, the enhancement of tensile properties due to bimodality has been reported $[1,15,16,22,23]$ but invariably for only unfilled system.

Looking at Figure 1 it is shown that the effect of bimodality on $\% \mathrm{~Eb}$ in presence of the $17.2 \%$ filler can be divided into three stages. In the first stage (0-90 mol\% P100), \% Eb gradually increases with increase of P100 to about 90 mole. In the second stage, the $\% \mathrm{~Eb}$ exhibits a dramatic increase with increase in P100 concentration up to $97 \mathrm{~mol} \%$. In the third stage, there is a drastic decrease with onward increase in P100 concentration.

The increase in UTS, $100 \%$ and elastic moduli with increase bimodality in the case of $17.2 \%$ filled network is shown as Figure 2. It is evident from this

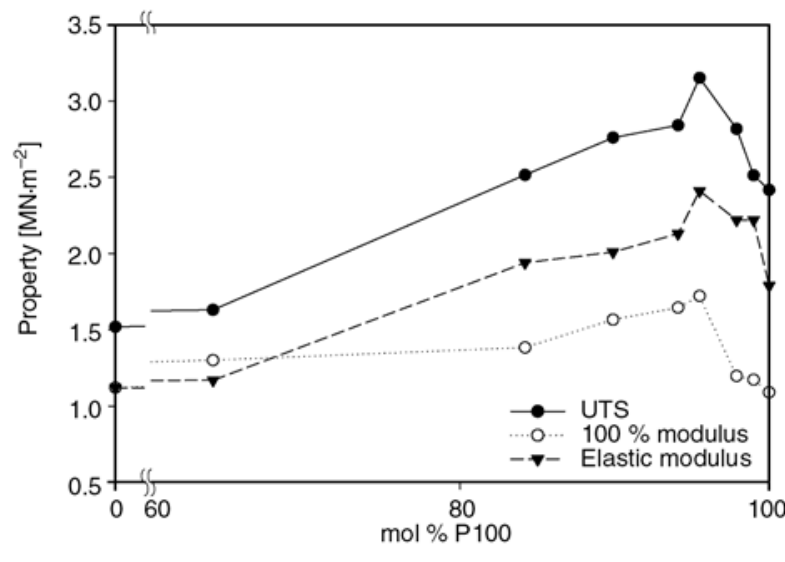

Figure 2. Effect of variation of bimodality on UTS, 100\% and elastic moduli

figure that the increase in all these three cases is almost linear and becomes optimum at about $95 \mathrm{~mol} \%$ short chain prepolymer. It is interesting that the optimum values of these properties in the case of unfilled bimodal silicone networks were observed and reported $[3,24]$ at $70 \mathrm{~mol} \%$ short chain prepolymer. This indicates that the incorporation of filler to bimodal silicone network shift the optimum for these mechanical properties to a higher limits of the short chain prepolymers. It is clear that the increase in UTS due to bimodality in the filled silicone system at about $95 \mathrm{~mol} \%$ short chain prepolymer as compared to the correspondingly filled monomodal network is about 2 fold. Similarly, in the case of $100 \%$ modulus as well as that of elastic modulus, an optimum increase of about 55 and $115 \%$ respectively can be noted due to 
bimodality. The incorporation of more than $95 \mathrm{~mol} \%$ short chain prepolymer with in the network, makes these properties inferior and a drastic decrease in these properties is observed. The effect of bimodality on the tensile properties of silicone networks in the presence of filler has not previously been reported.

The about 2 fold increase in UTS due to bimodality for the 17.2\% filled C50/P100 system can be attributed to the increase in the number of effective crosslinks with increase in short chain prepolymer up to $95 \mathrm{~mol} \%$. These crosslinks, whether chemical or physical, originate both from the short chain prepolymer and filler and tighten the chain network. The longer HOPDMS chains present in the bimodal networks at the same time retain the flexibility of the polymer chains. Unfortunately, in the case of highly filled $(30.2 \%)$ networks the contribution of effective crosslinks from the filler coupled with successive increases in short chain prepolymer tips the balance between chain tightening due to crosslinking and chain flexibility. As a result, the increase in concentration of short chain prepolymer decreases the elongation and reduces the UTS. As shown in Figure 3, the \% Eb shows a linear decrease with the increase in concentration of short chain HOPDMS. In the case of UTS, a gradual decrease is first recorded with increase in mol\% of P100 as shown in Figure 4 and then a drastic drop in the property occurs. The inferior $\% \mathrm{~Eb}$ and UTS of the $30.2 \%$ filled bimodal networks for all the compositions as compared to that of $17 \%$ filled one networks are due to the inability of the network to absorb the applied load reversibly. However in contrast, the elastic modulus for the $30.2 \%$ filled bimodal networks experiences a slow increase up

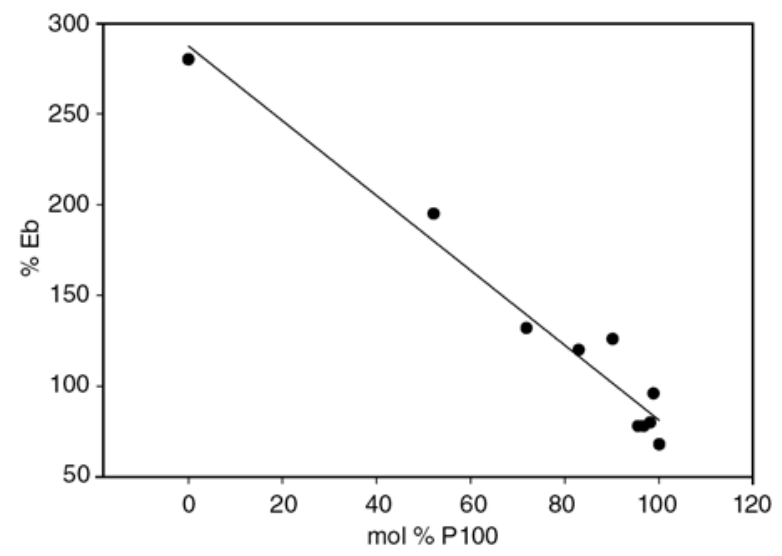

Figure 3. Effect of variation of bimodality on $\% \mathrm{~Eb}$ for $30.2 \%$ filled silicone networks

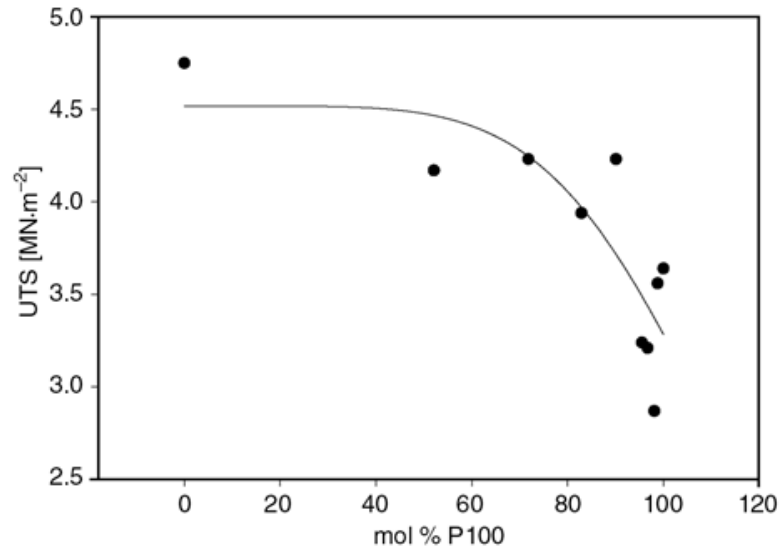

Figure 4. Effect of variation of UTS with mol\% P100 for $30.2 \%$ filled silicone networks

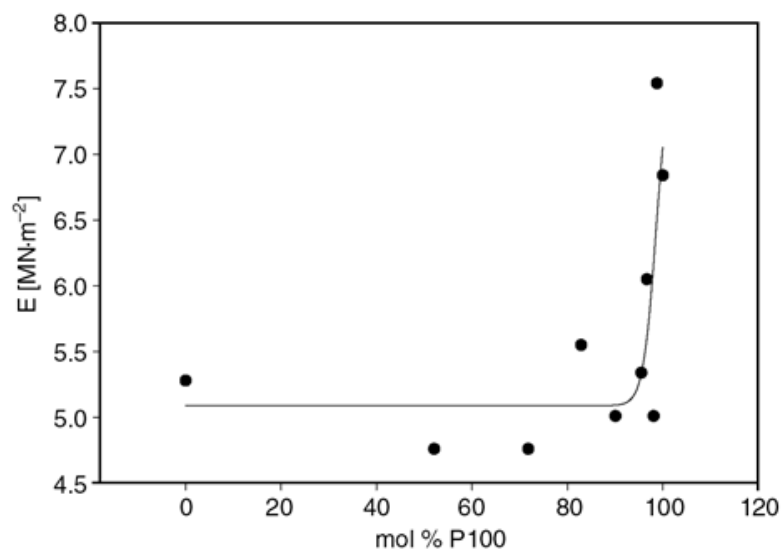

Figure 5. Effect of variation elastic modulus with mol\% P100 for $30.2 \%$ filled silicone networks

to about $80 \mathrm{~mol} \% \mathrm{P} 100$ followed by an exponential increase (Figure 5) with concentration of short chain HOPDMS. In this highly loaded (30.2\% filled) system the $\mathrm{OH}$ groups on the filler (2.5 to $3.5 / \mathrm{nm}^{2}$, surface area of filler $125-400 \mathrm{~m}^{2} / \mathrm{g}$ ) are more than sufficient to react via crosslinker with short chain prepolymer available and results in a network having inactive filler. In presence of sufficient fillers anchoring along the polymer chain at frequent interval, the further successive increments of short chain decrease the degree of freedom of orientation of polymer in the resulting networks. This employ that the excessive amounts of filler (30.2\%) coupled with the increase in amount of short chains some how undermines the role of the long chain prepolymer in the silicone network in reference to these mechanical properties. These inactive points may act as stress centers and hence responsible for the inferior tensile properties.

The enhancement in the tear, tensile and tensileoptic behaviour due to bimodality in the unfilled 
state of polydimethylsiloxanes has been dealt in detail in literature [7, 25-30]. A number of studies including experimental, numerical and analytical ones have been carried out to predict the said enhancement and ascertain its relationship with the various parameters of the structure of the bimodal polymer network.

For example von Lockette and Arruda [29] set a crosslinking algorithm, which apart from predicting the enhancement in mechanical properties, show correlation between the molar short chain concentration at which enhancement in the toughness occurs and the magnitude of certain short chain topologies peak, namely the density of loops in the gel and the percentage of doubled connections in the gel.

Von Lockette et al. [30] also developed a micromechanics-based constitutive model for the non-linear large deformation stress and birefringence responses of bimodal networks. This law is claimed to have capability of comparatively straightforward implementation to tensile test and tensile-optic behaviour of bimodal networks compared to the previous theories and moreover is predictive over a wide range of composition.

However, the above mentioned crosslinking algorithm and the micromechanics-based constitutive models are designed for unfilled bimodal polydimethylsiloxanes and unfortunately no such model exists presently for application to the present system.

The effect of filler on monomodal networks is also generally well documented [31-37]. The reinforcement of polymer network is related to the concentration of filler. The size and nature of the filler particle with in the polymer network determine the effective crosslinks which in turn are responsible for improvement in the mechanical properties [38]. For example, Bueche [39] reported an increase up to about 6 fold in effective crosslinks in polydimethylsiloxane on addition of $50 \mathrm{phr}$ silica. Polmanteer and Helmer [38] realizing the presence of this interaction have enumerated the various kinds of linkages that can be present in the filler vulcanisate. It is all these crosslinks which can be used to explain the increasing physical properties such as tensile strength with increase in concentration of filler.

Bueche suggests that the interaction between polymer and filler whether chemical or physical between the $\mathrm{Si}-\mathrm{O}$ of the resin with the hydrogen of the filler promotes reinforcement. It is well established $[40,41]$ that this reinforcement depends upon the chemical nature as well as on the size of the particles. A ten time increase in UTS and 100\% modulus and 2.5-5 times increase in \%Eb for silicone networks on incorporation of HDK (filler) has also been be attributed to the small particle size of the filler $(5-30 \mathrm{~nm})$ and high concentration of surface silanol groups $(2-3.5 \mathrm{SiOH} / \mathrm{nm})$.

The mechanical property of elastomers depends upon the number of effective linkages in the polymer network. These linkages are of different kinds and their identification becomes difficult after incorporation of filler in to the polymer network. However, the addition of filler considerably increases the effective crosslinks. The unexpected improvement in tensile properties due to bimodality in $17.3 \%$ filled silicone system is difficult to explain. In unfilled polymer system the effective crosslinks consists of: i) crosslink joining polymer with polymer and ii) polymer entanglement.

In the case of filled monomodal polymer system, the effective crosslinks constitute from the:

1) filler to polymer covalent linkage,

2) filler to polymer linkage resulting from polar or Van der Waals forces between filler and polymer,

3) effective filler to polymer linkage from entanglement influenced by filler structure,

4) effective filler to polymer linkage resulting from simple wetting of the filler surface by polymer.

In fact it is difficult to separate all these kinds of linkage from one another, analyse it and then attribute each one with a separate explanation. Then further more, explanation for improvement in the mechanical properties of filled (17.5\%) silicone system due to bimodality, becomes even more difficult.

In the case of highly filled (32.2\%) silicone networks, the $100 \%$ modulus records increase in its values up to about 84 mole\% of P100. Further increase in mole $\%$ of $\mathrm{P} 100$ results in polymer networks which fails prematurely during the test and hence this property can not be recorded. For modulus of elasticity, there is an increase in its values up to incorporation of about 98 mole\% P100 after which a decrease is observed. The data is given in Table 1. 


\section{Conclusions}

Like the tear property of unfilled and filled silicone network, the tensile behaviour of the filled silicone polymer network is sensitive to bimodality. The effect of bimodality on the tensile properties was also found to be beneficial for the $17.2 \%$ filled silicone network. The optimum in tensile properties was observed at $95 \mathrm{~mol} \%$ of short chain prepolymer. In the case of highly filled (32.2\%) networks bimodality brought adverse effect on the tensile properties such as UTS, \% Eb. In the light of the results of the present studies, the slightly higher physical properties of the high molecular weight prepolymer network reported earlier in the literature can be sacrificed in favour of the easy processing of low molecular silicone prepolymers in the low filled $(17.2 \%)$ bimodal networks.

\section{References}

[1] Andrady A. L., Llorente M. A., Mark J. E.: Model networks of end-linked polydimethylsiloxane chains. VII. Networks designed to demonstrate non-Gaussian effects related to limited chain extensibility. Journal of Chemical Physics, 72, 2282-2290 (1980).

[2] Bhowmick A. K., Stephens H. L.: Handbook of elastomers. New Developments and Technology. Marcel Dekker, New York (1988).

[3] Shah G. B., Winter R. W.: Effect of bimodality on tear properties of silicone networks. Macromolecular Chemistry and Physics, 197, 2201-2208 (1996).

[4] Galiatsatos V., Mark J. E.: Non-Gaussian optical properties of bimodal elastomeric networks. Macromolecules, 20, 2631-2632 (1987).

[5] Zeigler J. M., Fearon F. W. G.: Silicone base polymer science: A comprehensive resource. American Chemical Society, Washington (1990).

[6] Galiatsatos V., Subramanian P.R.: Stress-optical properties of bimodal networks. Macromolecular Symposia, 76, 233-240 (1993).

[7] Monnerie L., Besbes S., Ceremelli I., Bokobza L.: Molecular orientation in deformed bimodal networks 2. Fourier transform infrared measurements on polydimethylsiloxane networks and comparison with theory. Macromolecules, 28, 231-235 (1995).

[8] Bahar I., Erman B., Bokobza L., Monnerie L.: Molecular orientation in deformed bimodal networks. 1. theory. Macromolecules, 28, 225-231 (1995).

[9] Zhang Z-M., Mark J. E.: Model networks of endlinked polydimethylsiloxane chains. XIV. Stressstrain, thermoelastic, and birefringence measurements on the bimodal networks at very low temperatures. Journal of Polymer Science: Polymer Physics Edition, 20, 473-480 (1982).
[10] Lake G. J., Thomas A. G.: The strength of highly elastic materials. Proceedings of the Royal Society of London, A, 300, 108-119 (1967).

[11] Shah G. B.: Aspects of the polycondensation of hydroxyfunctional polydimethylsiloxanes. PhD Thesis, University of Wales, College of Cardiff, UK (1990).

[12] Wen J., Mark J. E.: Torsion studies of thermoelasticity and stress-strain isotherms of unimodal, bimodal, and filled networks of poly(dimethylsiloxane). Polymer Journal, 26, 151-157 (1994).

[13] Wada T., Ito K.: Heat-curable elastomeric silicones compositions. U.S. Patent 3652475, USA (1972).

[14] Bobear W. J.: Organopolysiloxane elastomer. U.S. Patent 3660345, USA (1972).

[15] Andrady A. L., Llorente M. A., Mark J. E.: Model networks of end-linked polydimethylsiloxane chains. IX. Gaussian, non-Gaussian, and ultimate properties of the trifunctional networks. Journal of Chemical Physics, 73, 1439-1445 (1980).

[16] Llorente M. A., Andrady A. L., Mark J. E.: Model networks of end-linked polydimethylsiloxane chains. XI. Use of very short network chains to improve ultimate properties. Journal of Polymer Science: Polymer Physics Edition, 19, 621-630 (1981).

[17] Patel S. K., Malone S., Cohen C., Gillmor J. R., Colby R. H.: Elastic modulus and equilibrium swelling of poly(dimethylsiloxane) networks. Macromolecules, 25, 5241-5251 (1992).

[18] Mark J. E., Andrady A. L.: Model networks of endlinked polydimethylsiloxane chains. X. Bimodal networks prepared in two-stage reactions designed to give high special heterogeneity. Rubber Chemistry and Technology, 54, 366-373 (1981).

[19] Yanyo L. C., Kelley F. N.: Effect of chain length distribution on the tearing energy of silicones elastomers. Rubber Chemistry and Technology, 60, 78-88 (1987).

[20] Grobler J. H. A., Mcgill W. J.: Effect of network heterogeneity on tensile and tear strengths of radiation, peroxide, efficient and conventional cured polyisoprene. Journal of Polymer Science Part A: Polymer Chemistry, 32, 287-295 (1994).

[21] Murphy J.: Additives of plastics handbook. Elsevier Advanced Technology, Oxford, UK (2001).

[22] Mark J. E.: The effect of strain-induced crystallization on the ultimate properties of an elastomeric polymer network. Polymer Engineering and Science, 19, 409 413 (1979).

[23] Erich F. R.: Rheology, vol 5., Academic Press, New York (1969).

[24] Shah G. B.: The effect of bimodality on the tear properties of filled silicone networks. Journal of Applied Polymer Science, 94, 1719-1722 (2004).

[25] Mark J. E., Tang M-Y.: Dependence of the elastomeric properties of bimodal networks on the length and amount of short chains. Journal of Polymer Science: Polymer Physics Edition, 22, 1849-1855 (1984). 
[26] Arruda E. M., Boyce M. C.: A three-dimensional constitutive model for the large stress behaviour of rubber elastic material. Journal of the Mechanics and Physics of Solids, 41, 389-412 (1993).

[27] Mark J. E.: The use of model polymer networks to elucidate molecular aspects of rubberlike elasticity. Advances in Polymer Science, 44, 1-26 (1982).

[28] Leung Y-K., Eichinger B. E.: Computer simulation of end linked elastomers 1 . Trifunctional network cured in the bulk. Journal of Chemical Physics, 80, 38773884 (1984).

[29] von Lockette P. R., Arruda E. M.: Topological studies of bimodal networks. Macromolecules, 32, 19901999 (1999).

[30] von Lockette P. R., Arruda E. M., Wang Y.: Mesoscale modeling of bimodal elastomer networks: Constitutive and optical theories and results. Macromolecules, 35, 7100-7109 (2002).

[31] Boonstara B. B., Cochrane H., Dannenberg E. M.: Reinforcement of silicone rubber by particulate silica. Rubber Chemistry and Technology, 48, 558-576 (1975).

[32] Smallwood W. M.: Limiting law of the reinforcement of rubber. Journal of Applied Physics, 15, 758-766 (1944).

[33] Bueche F.: Mullins effect and rubber-filler interaction. Journal of Applied Polymer Science, 5, 271-281 (1961).
[34] Kraus G.: Reinforcement of elastomers. Wiley, New York (1965).

[35] Sudár A., Móczó J., Vörös Gy., Pukánszky B.: The mechanism and kinetics of void formation and growth in particulate filled PE composites. Express Polymer Letters, 1, 763-772 (2007).

[36] Li Z. H., Zhang J., Chen S. J.: Effects of carbon blacks with various structures on vulcanization and reinforcement of filled ethylene-propylene-diene rubber. Express Polymer Letters, 2, 695-704 (2008).

[37] Kolesov I. S., Radusch H-J.: Multiple shape-memory behavior and thermal-mechanical properties of peroxide cross-linked blends of linear and short-chain branched polyethylenes. Express Polymer Letters, 2, 461-473 (2008).

[38] Polmanteer K. E., Helmer J. D.: Complexities of crosslink density in filled elastomers. Rubber Chemistry and Technology, 38, 123-133 (1965).

[39] Bueche A. M.: The curing of silicone rubber with benzoyl peroxide. Journal of Polymer Science, 15, 105120 (1955).

[40] Mark J. E.: Elastic properties of model polymer networks. Pure and Applied Chemistry, 53, 1495-1504 (1981).

[41] Gessler A.: Evidence for chemical interaction in carbon and polymer associations. Butyl and acidic oxy blacks. The possible role of carboxylic acid groups on the black. Rubber Chemistry and Technology, 42, 850-857 (1969). 